Evidence for Early Comprehension of Action Verbs

Iris Nomikou

University of Portsmouth, England

Katharina J. Rohlfing

Paderborn University and CITEC, Bielefeld University, Germany

Philipp Cimiano

CITEC, Bielefeld University, Germany

and

Jean M. Mandler

University of California, San Diego

Author Note

This study was part of the interdisciplinary project IP-13 on "Towards a model for the incremental co-development of linguistic and conceptual representation of actions" at the Cluster of Excellence Cognitive Interaction Technology “CITEC" (EXC 277), Bielefeld University funded by the German Research Foundation (DFG). We are grateful to the participating children and their parents, to Elena Justus for piloting the study and making the audio recordings, and to Marc Rohlfing and Malte Schilling for their data analysis scripts. We also wish to thank the readers of our poster at the ICIS 2016 for constructive discussions.

Correspondence concerning this article should be addressed to Iris Nomikou, University of Portsmouth, Department of Psychology, King Henry Building, King Henry I Street, Portsmouth, Hampshire, England PO1 2DY

E-mail: iris.nomikou@port.ac.uk 


\begin{abstract}
Applying an eye-tracking technique, we tested early verb understanding in 48 infants aged 9 and 10 months. Infants saw two objects presented side by side and heard a verb that referred to a common action with one of these objects (e.g., eating relating to a banana). The verbs were spoken by the parent in an interrogative manner in order to elicit a looking behavior in the infant. Results showed that 9-month-old infants did not show recognition of our test words. However, 10-month-old infants were able to understand a number of the tested verbs. In the discussion, we relate our findings to the nature of early verb representations.
\end{abstract}




\section{Evidence for Early Comprehension of Action Verbs}

Studies on early language acquisition reveal accumulating evidence suggesting that 6month-old infants already understand language way before they speak their first words. Notably, these studies focus on the acquisition of nouns. One of the first studies used a preferential looking procedure and found that infants as young as 6 months of age understood some nouns such as mommy or daddy that were familiar from their everyday experience (Tincoff \& Jusczyk, 1999). Follow-up studies revealed that at this early age, infants are also able to understand words referring to body parts such as hand and feet (Tincoff \& Jusczyk, 2012) that are commonly used in early games between caregivers and their children.

Bergelson and Swingley (2012) studied the comprehension of nouns in two types of categories: eight body parts (such as nose, mouth) and eight items related to food (such as spoon, banana). Using eye-tracking technology, they found that infants at the age of 6 months were not just capable of recognizing the correct item (e.g., banana) when presented paired on a screen with, for example, mouth. They could also select it from a more complex scene involving related objects on a table.

Evidently, young infants are capable of referential word understanding before they can produce words. This understanding is not just interesting with respect to building up a lexicon. It may also foster the segmentation of the speech stream: Bortfeld, Morgan, Golinkoff, and Rathbun (2005) found that infants recognized novel words following familiar - but not unfamiliar - names. This effect might depend on infants' knowledge of the meaning of the familiar word.

Up to now, however, most studies on word comprehension have focused on infants' early understanding of nouns. This is probably because researchers report that nouns are acquired earlier than verbs (e.g., Gentner, 1982; Gentner \& Boroditsky, 2001). Indeed, research has shown that very few verbs appear in the first 10 (Tardif et al., 2008) or 50 words comprehended in either English (Fenson et al., 1994) or German (Kauschke \& Hofmeister, 
2002). In other languages, it has been shown that verbs might occur earlier when that language provides a structure for their rich occurrence in input (see Au, Dapretto, \& Song, 1994, and Choi \& Gopnik, 1995, for Korean; Tardif, Gelman, \& Xu, 1999, for Mandarin).

Discussions on the development of semantics have considered whether early verbs differentiate between self-actions and observed actions. Investigating English-learning children, Huttenlocher, Smiley, and Charney (1983) conducted two studies: In a language comprehension study, 20- to 42-month-olds were presented with 10 sets of two contrasting videos and asked to select one on which, for example, a person is running (in contrast to walking). The authors found support for their hypothesis that verbs relating to movements were the easiest for the children to identify, whereas verbs relating to change of involved entities (such as clean or take) were more difficult. To assess early spontaneous production, the authors analyzed recordings of 23- to 28-month-olds in a variety of contexts (playground, store, and home). The data supported their proposed model of the development of action categories by showing that infants' verb meanings for self-actions are acquired prior to meanings for observed actions; and that young children seem to analyze the latter in terms of movement.

However, their model does not link up with findings presented in a more recent review by Sootsman Buresh, Woodward, and Brune (2006). This suggests that already:

by the end of the first year of life, infants have the conceptual material relevant for a range of verb meanings, including not only verbs that encode the observable trajectories of moving objects (e.g., fall) but also verbs that encode the outcomes of causal sequences (e.g., open), intentional actions (e.g., get), transactions (e.g., give), and psychological states (e.g., see). (p. 214)

Summarizing the literature on infants' understanding of actions as well as early verb learning, these authors concluded that it is not just the physical motions through space that infants perceive when seeing other persons acting. Instead, from early on, infants understand human 
actions as relational with respect to the performer(s), objects, and goals. However, the authors also identified a gap indicating that it takes children one year to move from action representation to its productive expression. Semantic development during this phase is not well understood.

The present study systematically investigated the age of acquisition of action verbs. We followed the approach presented by Mandler (2012), suggesting that early concepts capture the roles of objects - that is, what the objects do and what is done to them. Situated actions seem to be crucial for getting concepts about objects off the ground. This implies that actions themselves must be conceptualized early in development, and that these concepts form a solid basis for the acquisition of verbs. In addition, Heft (1989) postulates the relevance of bodily states that underlie the meaningful representations of entities.

In contrast to nouns - which some approaches would argue involve one-to-one formmeaning mappings - verbs are relational, and their meaning involves the perception of a situation involving agents, actions, objects, and the relationships between them. Even though children seem to use nouns before verbs, it is possible that action information is crucial; and that in their initial use, nouns "cumulate" situational elements (Werner \& Kaplan, 1963). For example, infants might say ball but relate it to the rolling ball (Nelson, 1974). Along similar lines, Samuelson, Smith, Perry, and Spencer (2011) found that children bind situational elements such as the location to an object's name.

Our study investigated 9- and 10-month-old infants using a technique similar to that of Bergelson and Swingley (2012), except that we used verbs in place of nouns. In fact, in a study following up their work on understanding nouns, Bergelson and Swingley (2013) published an article on early understanding of abstract words in 6- to 16-month-old infants: Among the pairs of words tested, three pairs consisted of action verbs (eat vs. hug, kiss vs. dance, and smile vs. drink); the others were mixed pairs (all gone vs. hi, more vs. splash, uhoh vs. bye, and wet vs. sleeping). These examples show that some of their tested words 
were more abstract than others, and the construction of the pairs did not focus on the syntactic role of the words. The results of this study revealed little understanding of any of the pairs by the 6- to 9-month-old group, and the verb pair that infants in the 10- to 13-month age range performed best on was kiss versus dance. The authors concluded that is not until the age of 14 to 16 months that evidence for understanding eat versus hug appears, although all seven pairs had positive scores. Thus, the data suggested tentatively that action verbs might be learned earlier than a variety of other word types such as all gone and hi. However, because the aim of the study was not to test action verbs alone, more work is needed to provide a clearer picture of the initial understanding of verbs.

Instead of using dynamic pictures as in Bergelson and Swingley (2013), we used static object pairs and instructed the parent to ask for the relevant verb. Our study extends the Bergelson and Swingley (2013) design by using a setup including only verbs contrasted across contexts in a systematic way. Moreover, we used a much narrower age range.

\section{Method}

\section{Participants}

We recruited 53 infants aged either 9 or 10 months. Five infants had to be excluded due to problems with calibration (2), hardware failure (1), and fussiness (2). Hence, the final sample contained 27 (16 female) 9-month-old ( $M=9 ; 7$ [months; days], $S D=13$ days $)$ and 21 (12 female) 10 -month-old infants $(M=10 ; 11 ; S D=11$ days). Infants were recruited from the Bielefeld area in Germany. Recruitment took place mostly through personal contact with midwives. Through these midwives, it was possible to access mother-infant courses and address the mothers personally, inform them about the study, gather their contact details, and also leave flyers for other interested families to contact us themselves. All participants were full-term healthy infants with no history of any hearing impairment and they heard $>80 \%$ German at home. In addition to participants' demographics, we obtained some information on infants' communicative as well as (loco)motor development via a parental survey that is 
currently under development (see Fischer, Nomikou, Grimminger, \& Rohlfing, 2018). For this study, we considered only the information related to the child's motor development. This is easy for parents to provide and therefore reliable. We simply asked whether or not the infants were already walking (regardless of whether this was in a supported or unsupported way).

\section{Materials}

Items. Motivated by the approach from the original study (Bergelson \& Swingley, 2012), we paired the verbal stimuli by drawing them from two different contexts: CARE and PLAY. The CARE context included the verbs essen [eat], schlafen [sleep], anziehen [dress], and baden [bathe] whereas the PLAY context consisted of the verbs bauen [build], sitzen [sit], lesen [read], and fahren [ride]. We created pairs contrasting verbs from the two contexts. The pairs were: eat-build, sleep-sit, dress-read, and bathe-ride. Table 1 shows that the verbs were selected to correspond to everyday activities that infants of this age can be expected to have experienced. ${ }^{1}$ In contrast to the original study, our word pairings did not present clear contrasts, because a child can, for example, play while being dressed or sleep while riding in a car.

\section{TABLE 1 HERE}

Pictures. The verbs were introduced while static images were being shown. We chose this method because a pilot study had revealed that infants have difficulty in watching two dynamic videos being presented side by side. Because Bergelson and Swingley (2015) found that infants showed comparable performance when presented with pictures versus short video

\footnotetext{
${ }^{1}$ In a pilot study, we asked parents whether they used these verbs with their infants. The verbs were confirmed as being common. Two further verbs were reported as being problematic: the verb to drink because parents reported that they often use the verb to eat for drinking milk or for nursing; the verb to swing because their infants did not have yet any experience with a swing. We therefore excluded these two items from the final verb selection.
} 
clips, we decided to use static images to present the verbs. The images showed everyday objects on a white background. Items were selected to be direct objects of the tested verbs (e.g., for the verb eat, we presented a picture of a banana). Pictures were retrieved from an online database (see Table 1). For each verb, we presented two different instantiations of the objects (e.g., a banana that is partly peeled and one that is not peeled). The understanding of a verb was tested twice, corresponding to the different instantiations appearing on the right or left side of the screen. In sum, each pair of objects appeared four times on the screen paired with one of two different verbs. Pictures were presented side by side on a 25 -inch LCD monitor, and the side of presentation was counterbalanced.

Audio. Motivated by the findings that 9-month-olds have a better semantic processing of nouns when the word is produced by their own mother (Parise \& Csibra, 2012), in our study, the stimuli were spoken by the parent. Parents were prompted over headphones that they wore during the experiment by hearing recordings of target sentences containing the investigated verbs. The recorded sentences were $1.5-2 \mathrm{~s}$ in duration and were presented at a low volume so that only the parent could hear them. The verbs were presented within a question. These questions were recorded in a recording booth by a native German-speaking woman. We designed the verbal presentation in such a way that the target verbs were in the sentence-final position. There were two different formats in which the target word was in an end position: The questions were: Magst Du X? [Do you like to X?] and Was kann man X? [What can you $\mathrm{X}$ ?], in which $\mathrm{X}$ stands for the target verb. One of these questions was presented for each item. The sentences were uttered in a child-directed way. The motivation for this kind of interrogative presentation was that (a) words in the sentence-end position have been reported to be easier to perceive (Goldfield, 1993), and (b) interrogatives have been reported to constitute a large proportion of maternal input in the first year of life (Soderstrom, Blossom, Foygel, \& Morgan, 2008).

\section{Procedure}


The study was approved by the local ethical committee of Bielefeld University. Parents and infants were met at the entrance of the building and escorted to the lab. Before the experiment began, the procedure was explained to them and they signed a consent form. During this time, the child had the opportunity to warm up by playing on the floor and exploring the room. After completing the consent form, both child and parent sat in front of the display and a folding screen was placed behind them, thus isolating them from the rest of the room. The infant sat on the parent's lap facing the display. Parents wore headphones to receive instructions and they also wore dark glasses to prevent them from seeing the screen. Data were collected using a Tobii X2-60 eye tracker.

\section{FIGURE 1 HERE}

Infants were presented with 16 paired-picture trials. The infants saw two images on the screen side by side: one from the CARE category and one from PLAY. Images were shown for a total of $9.5 \mathrm{~s}$. Within the first $3 \mathrm{~s}$ of each trial, parents heard a beep before the target sentence and then a second beep prompting them to begin repeating the sentence (see Figure 1). During sentence production, in which the parent was voicing the target verb in the sentence-end position (see section above on audio stimuli), the experimenter pressed a key on a wireless keyboard to mark the precise moment when the infant heard the verb. This was logged into the data. Due to individual differences in the parent's production of the target phrase (e.g., reaction time, or speed of uttering the sentence), slight variations in the length of time before the parent said the target word (verb) were expected. Participants were randomly assigned to one of five pseudorandomized trial orders that counterbalanced side, picture instance, and the ordering of images and target items.

After each trial, infants saw an attention getter. This was a 3-s clip featuring colorful animated shapes moving at the center of the screen accompanied by different sounds such as 
bells, coins, a car horn, and so forth. There were 15 different such clips that were also presented in five pseudorandomized orders. The experiment lasted $5 \mathrm{~min}$, after which families were asked to fill out a questionnaire containing demographic questions as well as questions on the child's communicative and motor skills (Fischer et al., 2018). Then a book was given to the infant as a present. The entire visit lasted about $45 \mathrm{~min}$.

\section{Analysis}

The posttarget analysis window extended from 367 to $4.500 \mathrm{~ms}$ after the onset of the spoken target word. For the onset of the target word, we used the recorded timestamp of the keyboard keypress and applied a Python script to split the looking times into two periods: before and after the uttered verb.

Word comprehension was measured by a difference score: the proportion of target looking upon hearing the target word (367 to 4,500 ms post keyboard keypress) minus the proportion of target looking before hearing the word (from when pictures were displayed until just before the keyboard keypress). This gives us a difference score value that can be either positive or negative. If the value is positive, it indicates that infants showed an increase of looking at the correct object after hearing the target verb. Note that, as in the original study (Bergelson \& Swingley, 2012), the four item pairs were fixed. The pairing allowed us to control for fixation preferences, because the same pair was presented visually to test the understanding of two different verbs. Instead of measurements of looking times at the target versus distractor, we took gaze at the target before and after the verb presentation into account and could, thus, mitigate the possibility of a particular item being looked at merely because of its appearance.

\section{Results}


We initially analyzed the timing of the keypress events recorded by the experimenter via the keyboard. This revealed that infants saw the presented pictures for an average of $4.8 \mathrm{~s}$ after the parent had spoken the target word $(M=4.76, S D=.48)$. Accordingly, we set the fixed time window at 4,500 $\mathrm{ms}$ after the keypress.

\section{FIGURE 2 about HERE}

We conducted a mixed, between, and within-subjects ANOVA ${ }^{2}$ with AGE (9 months vs. 10 months old) as the between-subjects variable, and TIME (before vs. after the word was spoken) as the within-subjects variable. We found a significant AGE x TIME interaction effect, $F(1,46)=5.687, p<.021, \eta^{2}=.107$. We also found a significant main effect of AGE, $F(1,46)=7.355, p<.009, \eta^{2}=.138$; and the post hoc pairwise comparisons with Bonferroni correction revealed a significant difference between 9- and 10-month-old infants, $t(46)=$ 2.712, $p<.01$. We did not find a significant effect of TIME, $F(1,46)=1.251, p<.269, \eta^{2}=$ .024. Additionally, an independent-samples $t$ test indicated that the difference scores were significantly higher for the 10 -month-olds $(M=.054, S D=.12)$ than the 9 -month-olds $(M=-$ $.015, S D=.09), t(46)=2.295, p=.026, d=0.66$. We therefore analyzed the data in separate groups. We additionally calculated a linear regression with the increase in looking times at the target as the dependent variable and infants' age in days as an independent variable. The regression model did not attain significance, suggesting that the change in performance was not linear, $F(1,46)=2.23, p=.142$.

\section{Nine-Month-Old Infants}

\footnotetext{
2 The assumption of normality was assessed with the Shapiro-Wilk test. The statistics for both the 9-month-olds $(S W=.956, d f=27, p=.292)$ and the 10 -month-olds $(S W=.913, d f=21, p=.063)$ suggested that normality was a reasonable assumption.
} 
On average, these infants spent $51.1 \%(S D=.056, M I N=36.7 \%, M A X=63 \%)$ of their looking time on the target object before the target word was spoken and $49.6 \%(S D=$ $.063, M I N=35.4 \%, M A X=62 \%$ ) of their looking time on the target object after the word had been spoken.

There were no differences between male $(M=-.047, S D=.08)$ and female participants $(M=-.008, S D=.09), t(25)=1.582, p=.126$. We found that 13 out of 27 (48.1\%) of the 9-month-olds' mean looking scores were positive. This proportion was not statistically significant according to a binomial test $(p=1.0)$. This result suggests that after the parent spoke the sentence containing the target verb, 9-month-olds did not orient toward the target object in any systematic way. Infants showed positive performance on two out of eight items. Averaged over all items, a one-sample $t$ test revealed no significant increase in looking time between before and after the verb was spoken by their parent $(M=-.015, S D=$ $.09, t(26)=-.848, p=.404)$.

\section{Ten-Month-Old Infants}

On average, these infants spent $43.4 \%(S D=.095, M I N=25.9 \%, M A X=60.1 \%)$ of their looking time on the target object before the target word was spoken and $49.6 \%(S D=$ $.081, M I N=31 \%, M A X=64.1 \%$ ) of their looking time on the target object after the word had been spoken. Again, no differences were found between male $(M=.064, S D=.09)$ and female participants $(M=.046, S D=.14), t(19)=-.331, p=.744$. We found that 17 out of 21 (81\%) 10-month-old infants showed positive performance in looking at the target object. According to a binomial test $(p=.007)$, infants in this age group significantly increased their gaze toward the target after hearing the target verb spoken by their parent. Infants showed positive performance on seven out of eight items (see Figure 3). Averaged over all items, a one-sample $t$ test showed a significant increase in looking time between before and after the verb was spoken by their parent $(M=0.054, S D=.12), t(20)=2.120, p=.047, d=0.45$. 


\section{FIGURE 3 HERE}

\section{Discussion}

This study extends existing research suggesting that improvement in verb understanding begins around 10 months by analyzing responses to verbs in 9- and 10-monthold infants. We used a preferential looking paradigm with an eye tracker to investigate whether infants can infer the meaning of verbs when presented with pictures of everyday objects as a potential referent while hearing sentences including a verb spoken in an interrogative manner by their parents. We used static images of object pairs in which only one object corresponded to the uttered verb (e.g., a book, but not trousers, corresponding to read). We measured the amount of infants' looking time toward the object before and after the target verb was spoken and calculated a proportion of looking at the target by using a difference score. An increase in fixation on the target picture after hearing the verb was taken as an indication for verb understanding. Using this technique, we studied eight verbs from the contexts CARE and PLAY.

We found significant differences in performance between 10- and 9-month-old infants. Therefore, we analyzed the two groups separately. Whereas $81 \%$ of the 10 -month-old infants showed an increase in target gaze for almost all presented verbs, performance of the 9month-old infants was not robust. This confirms findings from previous research indicating that at 10-13 months, infants are starting to understand abstract words (Bergelson \& Swingley, 2013). Our findings also extend these findings in three ways: First, we could narrow down the age window from $10-13$ months $(M=11.96$ months) to 10 months of age. Second, we could show that a significant developmental change happens between 9 and 10 months of age. Third, we could show that infants can alter their attention toward object stimuli when hearing verbs.

With respect to the age difference, the data obtained via parental survey on the infants' self-locomotion skills suggest that factors other than age alone could be linked to the 
demonstrated verb understanding. According to the parental survey, only three of the 10month-olds in our sample were not (supported or unsupported) walkers, indicating that between 9 and 10 months of age one significant development seems to be in self-locomotion skills. This finding is also corroborated by our regression analysis on the relationship between age and verb understanding that seems to indicate no gradual increase in performance, but rather a more abrupt change. The fact that most of the 10-month-olds in our study had walking experience speaks to a link between the development of language and motor skills (He, Walle, \& Campos, 2015). We propose that related to walking skills, a gain in interactional experience might also be at play. More specifically, when children start to walk, the verbal responses from their mothers change. In this vein, Karasik, Tamis-LeMonda \& Adolph (2014) found that mothers of walkers provide more action directives than mothers of crawlers. Consequently, infants might receive verbal input that relates directly to the object's actions and thus shapes infants' early verb understanding. Future work is needed to shed more light on this relationship between motor and language development.

The data from our experiment say little about the meaning that the infants have acquired when showing their understanding. Clearly, they have begun to associate some form of meaning, but the question whether children look at a banana when they hear the word essen [eat], because they know what this action means, remains unanswered. Associations have been proposed as a start in verb learning (Childers, Bottera, \& Howard, 2018). The forms of associations revealed in our experiment speak to the possibility that verbs bind not only aspects of actions but also characteristics of objects that are related to these verbs. In other words, our findings suggest that 10-month-old infants can associate verbs to object stimuli, suggesting that action concepts can be evoked in object perception (Glenberg \& Gallese, 2012; Mandler, 2012). This complements research proposing that children learn language by building relations and drawing from rich concepts that need to be refined in order to converge with the linguistic categories that a target language entails (Bowerman \& Choi, 2003; 
Göksun, Hirsh-Pasek, \& Golinkoff, 2010; Pulverman, Song, Hirsh-Pasek, Pruden, \& Golinkoff, 2013).

It is also possible that the distinction between nouns versus verbs does not account for the way young children encounter situations - an idea Huttenlocher and Smiley (1987) already discussed in relation to the early meanings of nouns. Instead of adult-like extensions of words, early meanings could capture "complexive" attributes of situations, "including the actions and locations of objects, etc." (Huttenlocher \& Smiley, 1987, p. 63). Because our stimuli were static images of objects, we can relate our results directly to findings reported by Huttenlocher and Smiley (1987) and we can suggest that verb understanding is complexive.

The proposition that objects are evoked in children when hearing a verb can have at least two explanations: One takes children's growing linguistic experience into account. Here, infants might have learned that the presented verb has arguments of certain sorts (the direct objects of eating), and that, for example, a banana is such an object. The existence of cognitive operations that are relevant for linguistic meaning has been recently reported for 14month-olds: Yin and Csibra (2015) found that when learning a new label, infants can form role-governed categories (being the chaser in a scene) that override perceptual form similarities. This finding suggests that infants use relational properties instead of one-to-one mappings to learn new words. In another study, Ferry, Hespos, and Gentner (2015) showed that analogical ability - the ability to make relational comparisons - is present in 7- and 9month-olds, suggesting the effectiveness of relational conceptual mechanisms before the production of verbs. Finally, evidence for the foundations of verb learning has been presented by Song, Pruden, Golinkoff, and Hirsh-Pasek (2016) who showed that 10- to 12-month-olds can categorize aspects of events such as path or manner as well as the role of actors in events. These findings attest to the infants' increasing ability to differentiate between types of motion verbs. 
A second explanation might target an associative meaning that can be achieved when children predict and mentally recall characteristic movements as well as the type of environmental change. Both aspects can be derived from the relation between the "initiator and a particular kind of object" (Huttenlocher et al., 1983, p. 76) that are used canonically for this action when they hear the sound of the verb-an achievement that has to do with retrieving knowledge from rich representations (Nomikou, Schilling, Heller, \& Rohlfing, 2016). In this case, we would need to know more about how the rich memories that the children seem to possess have become "stripped" from the situational context and thus “semanticized" over time (Ofen \& Shing, 2013, p. 2261).

Future work can test these explanations by selecting objects that are related to eating but are not direct objects (e.g., a spoon or a plate rather than something to eat). We assume that if the effects prove to be similar to those for the banana, they would support the associative meaning explanation, whereas other effects would probably support the linguistic experience explanation. However, as parents tend to expand their utterances toward children and to use more lexical information with age, it is possible that children will increasingly hear spoon in the context of eating.

Regarding the research on the ability of infants to categorize aspects of actions, our study adds the important finding that infants as young as 10 months are already on their way to understanding aspects of verbs when these are presented to them by their parent in an interrogative manner. Thus, studies on the discrimination of actions in early development (Song et al., 2016) that suggest a period of prelinguistic categorization need to take into account that a linguistic influence is already in play at an early age (Nomikou et al., 2016). The present work shows that infants begin to understand verbs earlier than typically claimed in the literature. 


\section{References}

Au, T. K. F., Dapretto, M., \& Song, Y. K. (1994). Input vs constraints: Early word acquisition in Korean and English. Journal of Memory and Language, 33(5), 567-582.

Bergelson, E., \& Swingley, D. (2012). At 6-9 months, human infants know the meanings of many common nouns. PNAS, 109, 3253-3258.

Bergelson, E., \& Swingley, D. (2013). The acquisition of abstract words by young infants. Cognition, 127(3), 391-397.

Bergelson, E., \& Swingley, D. (2015). Early word comprehension in infants: Replication and extension. Language Learning and Development, 11(4), 369-380.

Bortfeld, H., Morgan, J. L., Golinkoff, R. M., \& Rathbun, K. (2005). Mommy and me: Familiar names help launch babies into speech-stream segmentation. Psychological Science, 16(4), 298-304.

Bowerman, M., \& Choi, S. (2003). Space under construction: Language-specific spatial categorization in first language acquisition. Language in mind: Advances in the study of language and cognition, 387-428.

Childers, J. B., Bottera, A., \& Howard, T. (2018). Learning how speakers use words to refer to actions. In G. Westermann \& N. Mani (Eds.), Early word learning (pp. 70-82). London, England: Routledge.

Choi, S., \& Gopnik, A. (1995). Early acquisition of verbs in Korean: A cross-linguistic study. Journal of Child Language, 22(3), 497-529.

Fenson, L., Dale, P. S., Reznick, J. S., Bates, E., Thal, D. J., Pethick, S. J., ... \& Stiles, J. (1994). Variability in early communicative development. Monographs of the Society for Research in Child Development, 59(5), v-173.

Ferry, A. L., Hespos, S. J., \& Gentner, D. (2015). Prelinguistic relational concepts: Investigating analogical processing in infants. Child Development, 86(5), 1386-1405. 
Fischer, S., Nomikou, I., Grimminger, A., \& Rohlfing, K. J. (2018, January). A parental questionnaire for infants' early communication abilities at the age of 8-10 monthsthe German "Baby-Komm." Poster presented at the BCCCD, Budapest, Hungary.

Gentner, D. (1982). Why nouns are learned before verbs: Linguistic relativity versus natural partitioning. Language, 2, 301-334.

Gentner, D., \& Boroditsky, L. (2001). Individuation, relativity, and early word learning. In M. Bowerman \& S. Levinson (Eds.), Language acquisition and conceptual development (pp. 215-256). Cambridge, England: Cambridge University Press.

Glenberg, A. M., \& Gallese, V. (2012). Action-based language: A theory of language acquisition, comprehension, and production. Cortex, 48(7), 905-922.

Göksun, T., Hirsh-Pasek, K., \& Golinkoff, R. M. (2010). Trading spaces carving up events for learning language. Perspectives on Psychological Science, 5(1), 33-42.

Goldfield, B. A. (1993). Noun bias in maternal speech to one-year-olds. Journal of Child Language, 20(1), 85-99.

He, M., Walle, E. A., \& Campos, J. J. (2015). A cross-national investigation of the relation between infant walking and language development. Infancy, 20, 283-305.

Heft, H. (1989). Affordances and the body: An intentional analysis of Gibson's ecological approach to visual perception. Journal for the Theory of Social Behaviour, 19(1), 1-30.

Huttenlocher, J., \& Smiley, P. (1987). Early word meanings: The case of object names. Cognitive Psychology, 19(1), 63-89.

Huttenlocher, J., Smiley, P., \& Charney, R. (1983). Emergence of action categories in the child: Evidence from verb meanings. Psychological Review, 90(1), 72-93.

Karasik, L. B., Tamis-LeMonda, C. S., \& Adolph, K. E. (2014). Crawling and walking infants elicit different verbal responses from mothers. Developmental Science, 17(3), 388-395. 
Kauschke, C., \& Hofmeister, C. (2002). Early lexical development in German: A study on vocabulary growth and vocabulary composition during the second and third year of life. Journal of Child Language, 29(4), 735-757.

Mandler, J. M. (2012). On the spatial foundations of the conceptual system and its enrichment. Cognitive Science, 36, 421-451.

Nelson, K. (1974). Concept, word, and sentence: Interrelations in acquisition and development. Psychological Review, 81, 267-285.

Nomikou, I., Schilling, M., Heller, V., \& Rohlfing, K. J. (2016). Language at all times: Action and interaction as contexts for enriching representations. Interaction Studies, 17(1), 128153.

Ofen, N., \& Shing, Y. L. (2013). From perception to memory: Changes in memory systems across the lifespan. Neuroscience \& Biobehavioral Reviews, 37(9), 2258-2267.

Parise, E. \& Csibra, G. (2012). Electrophysiological evidence for the understanding of maternal speech by 9-month-old infants. Psychological Science, 23, 128-733.

Pulverman, R., Song, L., Hirsh-Pasek, K., Pruden, S. M., \& Golinkoff, R. M. (2013). Preverbal infants' attention to manner and path: Foundations for learning relational terms. Child Development, 84(1), 241-252.

Samuelson, L. K., Smith, L. B., Perry, L. K., \& Spencer, J. P. (2011). Grounding word learning in space. PloS One, 6(12), e28095.

Soderstrom, M., Blossom, M., Foygel, R., \& Morgan, J. L. (2008). Acoustical cues and grammatical units in speech to two preverbal infants. Journal of Child Language, 35(4), 869-902.

Song, L., Pruden, S. M., Golinkoff, R. M., \& Hirsh-Pasek, K. (2016). Prelinguistic foundations of verb learning: Infants discriminate and categorize dynamic human actions. Journal of Experimental Child Psychology, 151, 77-95. 
Sootsman Buresh, J., Woodward, A., \& Brune, C. W. (2006). The roots of verbs in prelinguistic action knowledge. In K. Hirsh-Pasek \& R. M. Golinkoff (Eds.), Action meets word. How children learn verbs (pp. 208-227). New York, NY: Oxford University Press.

Tardif, T., Fletcher, P., Liang, W., Zhang, Z., Kaciroti, N., \& Marchman, V. A. (2008).

Baby’s first 10 words. Developmental Psychology, 44(4), 929-938.

Tardif, T., Gelman, S. A., \& Xu, F. (1999). Putting the "noun bias" in context: A comparison of English and Mandarin. Child Development, 70(3), 620-635.

Tincoff, R., \& Jusczyk, P. W. (1999). Some beginnings of word comprehension in 6-montholds. Psychological Science 10(2), 172-175.

Tincoff, R., \& Jusczyk, P. W. (2012). Six-month-olds comprehend words that refer to parts of the body. Infancy, 17(4), 432-444.

Werner, H., \& Kaplan, B. (1963). Symbol formation: An organismic-developmental approach to language and the expression of thought. New York, NY: Wiley.

Yin, J., \& Csibra, G. (2015). Concept-based word learning in human infants. Psychological Science, 26(8), 1316-1324. 
Table 1

Target Verb Pairings in Their Different Contexts

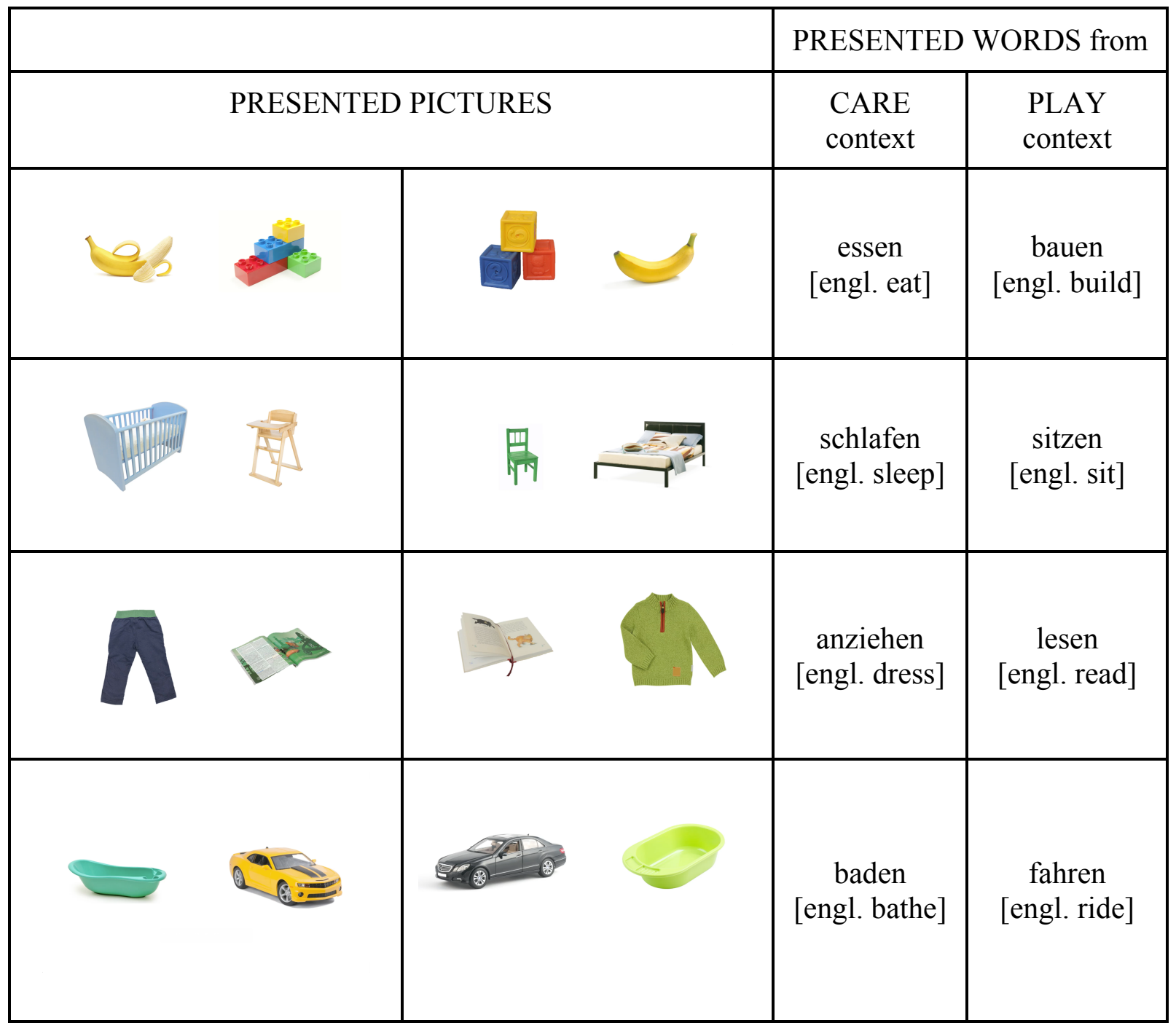




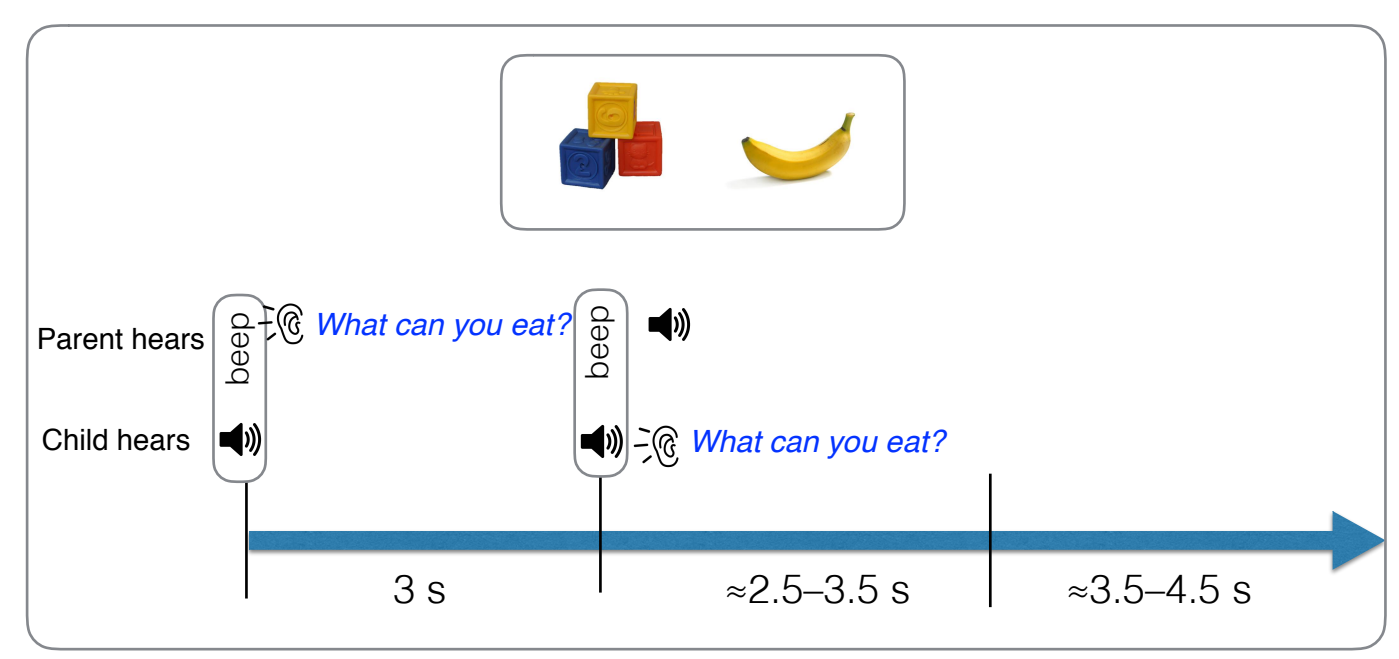

Figure 1. Timing of audio and visual presentation. The first time window in which the parent heard the phrases over the headphones was fixed. The two intervals following the second beep are averages, because the time taken to speak the sentence varied from parent to parent. 

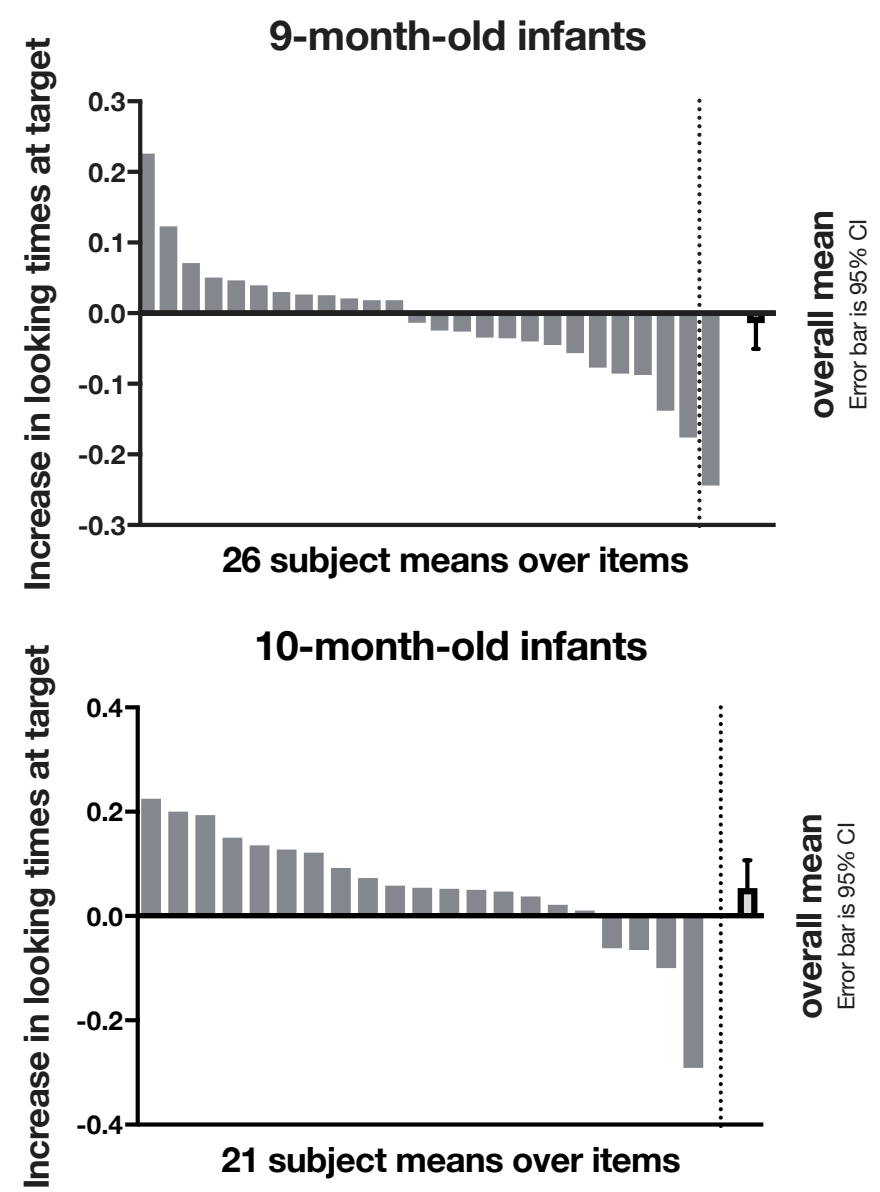

Figure 2. Individual subjects' increase in gaze at target averaged across items in the two age groups. 

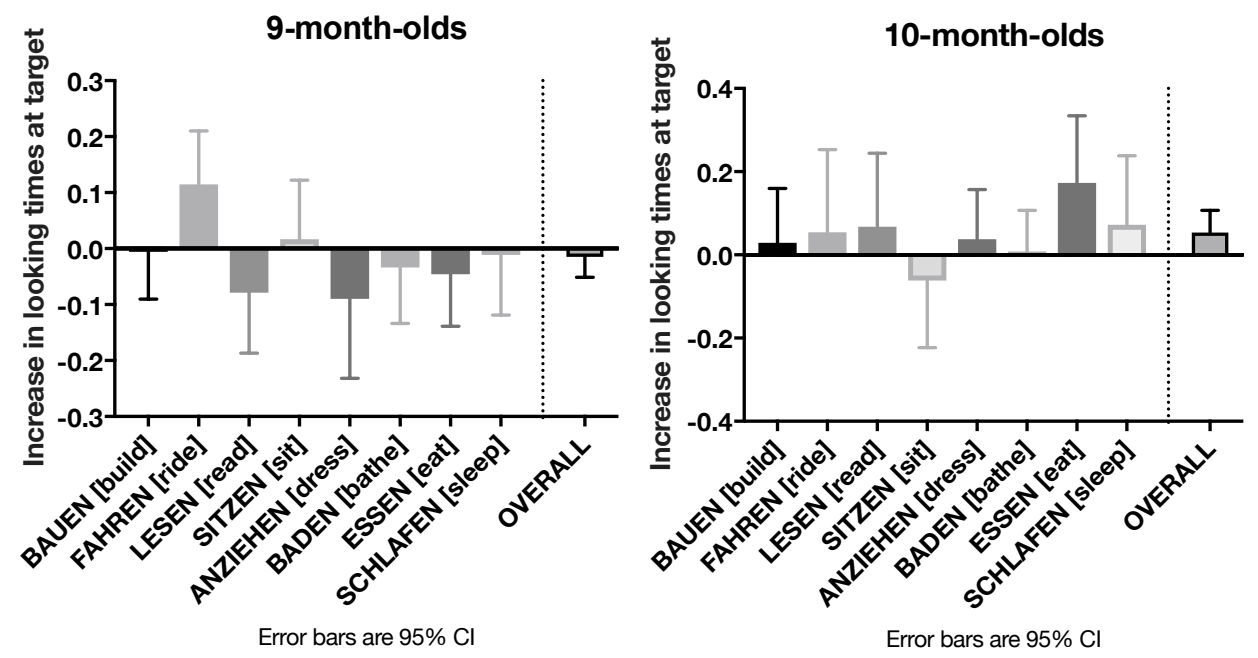

Figure 3. Infants' increase in gaze at target upon hearing the target word for all individual items in the two age groups. The target word was drawn from two different contexts: PLAY (left) and CARE (right). 